\title{
A New Species of Geranium Sect. Neoandina (Geraniaceae) from Colombia
}

Author(s): Carlos Aedo

Source: The Journal of the Torrey Botanical Society, 134(4):534-539. 2007.

Published By: Torrey Botanical Society

DOI: http://dx.doi.org/10.3159/07-RA-031.1

URL: http://www.bioone.org/doi/full/10.3159/07-RA-031.1

BioOne (www.bioone.org) is a nonprofit, online aggregation of core research in the biological, ecological, and environmental sciences. BioOne provides a sustainable online platform for over 170 journals and books published by nonprofit societies, associations, museums, institutions, and presses.

Your use of this PDF, the BioOne Web site, and all posted and associated content indicates your acceptance of BioOne's Terms of Use, available at www.bioone.org/page/ terms_of use.

Usage of BioOne content is strictly limited to personal, educational, and non-commercial use. Commercial inquiries or rights and permissions requests should be directed to the individual publisher as copyright holder. 


\title{
A new species of Geranium sect. Neoandina (Geraniaceae) from Colombia ${ }^{1}$
}

\author{
Carlos Aedo ${ }^{2,3}$ \\ Real Jardín Botánico, Consejo Superior de Investigaciones Científicas, Plaza de Murillo 2, \\ 28014 Madrid, Spain
}

\begin{abstract}
Aedo, C. (Real Jardín Botánico, Consejo Superior de Investigaciones Científicas, Plaza de Murillo 2 , 28014 Madrid, Spain). A new species of Geranium sect. Neoandina (Geraniaceae) from Colombia. J. Torrey Bot. Soc. 134: 534-539. 2007.-A new species, Geranium alonsoi, from Boyacá and Cundinamarca in Colombia is described and illustrated, and the key to the species of Geranium sect. Neoandina is upgraded.
\end{abstract}

Key words: Geraniaceae, Geranium, Colombia, taxonomy.

The genus Geranium L. comprises about 380 species distributed throughout most of the world. South America is the richest area of the world, with over 130 species. Most of these species belong in subg. Geranium. The exceptions are section Brasiliensia R. Knuth (Aedo 2001a) included in subg. Erodioidea (Picard) Yeo and some non-native representatives of subg. Robertium (Picard) Rouy (Aedo et al. 1998). The genus was monographed by Knuth (1912), but no recent revisions for South America are available. Aedo (2000, 2001b) revised Geranium in North America and Moore (1943) in Central America, but there are no native species in these revisions that occur south of Panama.

In pursuit of my aim to prepare a comprehensive monograph of the genus I have studied some groups of Geranium from South America (Aedo 2001a, Aedo et al. 2002, 2003, 2005). One of these taxonomic revisions was that of Geranium sect. Neoandina Aedo resulting in the recognition of 22 species (Aedo et al. 2002) of this section, plus one species of sect. Azorelloida Aedo \& al., and two other of sect. Paramensia R. Knuth. Soon afterwards a new species endemic from Peru was added to sect. Neoandina (Aedo 2004). In this paper, a

\footnotetext{
1 This work was partly financed by the Spanish Government through research project CLG200400172.

2 The author wish to thank M. Laínz for the Latin diagnosis, and S. Castroviejo for uncompromising support. I also thank the editor and two anonymous reviewers for helpful comments. The following herbaria are thanked for loans or access to material: COL, F, GH, HUA, MICH, MO, NY.

3 E-mail: aedo@rjb.csic.es

Received for publication July 9, 2007, and in revised form September 28, 2007.
}

new species of this section is described from unidentified specimens of Geranium.

Geranium alonsoi Aedo, sp. nov. TYPE: Colombia. Cundinamarca, Villapinzón, de Villapinzón a Umbita, 21 May 1998, Fernández Alonso et al. 15573 (holotype, MA-641407; isotypes, COL! HUA!)

A simili Geranium sibbaldioides Benth. praecipue differt cymulis 2-4-plo longioribus quam adiacenti folio -in hoc autem cymulae nunquam 2-plo longiores quam adiacens folium.

Herbs 20-69 cm tall, perennial, procumbent. Rootstock 0.9-2.7 mm diam., \pm vertical; with short vegetative stems. Leaf lamina 0.9 $2.1 \mathrm{~cm}$ long, $1.3-2.3 \mathrm{~cm}$ wide, polygonal in outline, cordate, palmatifid (divided for 0.80 0.94 of its length), usually glabrous on both sides, with antrorse, eglandular cilia 0.1 $0.2 \mathrm{~mm}$ long on the margin, each segment ending in 1-3 bristles $0.3-0.8 \mathrm{~mm}$ long, sometimes \pm hairy on abaxial surface, not coriaceous ( \pm herbaceus); segments 5 , lanceolate, sometimes obtriangular (with lanceolate lobes), $0.7-1.2 \mathrm{~mm}$ wide at the base, 1(3)lobed at the apex (ratio main-sinus length of the middle segment $/$ middle-segment length $=$ $0.46-0.58$ ); petioles to $2.7 \mathrm{~cm}$ long, with retrorse, appressed, eglandular hairs 0.1 $0.4 \mathrm{~mm}$ long; stipules $7.5-11.7 \mathrm{~mm}$ long, 1.1 $2.5 \mathrm{~mm}$ wide, lanceolate (with a setaceous apex $2.8-4.3 \mathrm{~mm}$ long), papery, reddish, with eglandular hairs on abaxial surface and on the margin, glabrous adaxially, ending in 1-3 bristles $0.4-0.8 \mathrm{~mm}$ long. Inflorescence in 1flowered cymules; peduncles $11-34.9 \mathrm{~mm}$, with retrorse, appressed, eglandular hairs $0.1-0.4 \mathrm{~mm}$ long; bracteoles 5-9.2 $\mathrm{mm}$ long, $0.7-1.2 \mathrm{~mm}$ wide; pedicels $21-80 \mathrm{~cm}$ long, with retrorse, appressed, eglandular hairs 
0.1-0.4 mm long; ratio cymule length /leaf length $=(1.9) 2.6-4.2$. Sepals $5-8.3 \mathrm{~mm}$ long, 1.9-2.8 mm wide (ratio pedicel length/sepal length $=4.2-13.1$ ), 3-nerved, mucronate (with mucro $0.4-1.2 \mathrm{~mm}$ long), with scarious margins $0.1-0.4 \mathrm{~mm}$ wide, with scattered erectpatent, eglandular hairs $0.4-1.2 \mathrm{~mm}$ long on base of abaxial side and margin, minutely hairy adaxially, ending in 1-3 bristles 0.4 $0.8 \mathrm{~mm}$ long. Petals $7.6-14.1 \mathrm{~mm}$ long, 3.4 $7.3 \mathrm{~mm}$ wide (ratio petal length/petal width $=$ 1.6-2.6), obovate, entire, without claw, hairy on both sides (mainly on the base of adaxial side), ciliate on the basal margin, with hairs $0.2-0.38 \mathrm{~mm}$ long, purplish. Filaments $2.8-$ $4 \mathrm{~mm}$ long, not exserted, lanceolate, glabrous, or with few eglandular hairs $0.2-0.4 \mathrm{~mm}$ long on the abaxial side and margin; anthers $0.8-$ $1.1 \mathrm{~mm}$ long, $0.6-1 \mathrm{~mm}$ wide. Nectaries glabrous. Gynoecium 3.8-6.1 mm long. Fruit $10.6-14.8 \mathrm{~mm}$ long; mericarps $2.2-3.3 \mathrm{~mm}$ long, $1.2-1.7 \mathrm{~mm}$ wide, with antrorse, \pm appressed, eglandular hairs $0.1-0.3 \mathrm{~mm}$ long; rostrum $6.4-9.4 \mathrm{~mm}$ long, without a narrowed, with erect-patent, eglandular hairs $0.2-0.3 \mathrm{~mm}$ long; stigmatic remains $1.2-$ $2.1 \mathrm{~mm}$ long (ratio fruit length/stigmatic remains length $=6.4-8.3$ ), with 5 glabrous lobes. Seeds $1.5-2 \mathrm{~mm}$ long, $0.9-1.3 \mathrm{~mm}$ wide; hilum 1/6 as long as the perimeter (Fig. 1).

Habitat and phenology. Paramo, in moss on wet sites along water courses or boggy areas dominated by shrubs and frailejons, 2900$3700 \mathrm{~m}$. Flowering between May and October.

Distribution. Colombia (departments of Boyacá and Cundinamarca). Fig. 2.

Paratypes. BoyACÁ: Duitama, páramo de la Rusia, $5^{\circ} 40^{\prime} \mathrm{N}, 73^{\circ} 5^{\prime} \mathrm{W}, 14$ May 1982 , Arrieta \& Castillo 82 (COL), Rodríguez \& Rojas 47 (COL). Gambita, vereda el Carmen, páramo de la Rusia, $5^{\circ} 40^{\prime} \mathrm{N}, 73^{\circ} 5^{\prime} \mathrm{W}, 14$ May 1982, Valbuena \& Harker 50 (COL). Machetá, Páramo de Güina, $5^{\circ} 5^{\prime} \mathrm{N}, 73^{\circ} 36^{\prime}$ W, 2 Oct 1986, Rangel et al. 4069 (MA). Páramo de Bijagual, $5^{\circ} 28^{\prime} \mathrm{N}, 73^{\circ} 11^{\prime} \mathrm{W}, 16$ Oct 1963, Espinal \& Montenegro 1375 (COL). Páramo de Huina entre Belén y Susacón, $6^{\circ}$ $10^{\prime} \mathrm{N}, 72^{\circ} 46^{\prime} \mathrm{W}, 6$ May 1959, Barclay 7582 (COL), Barclay \& Juajibioy 7630 (COL). Páramo de la Rusia near La Osera, station 83 along road between Duitama and Charalá, $5^{\circ} 40^{\prime} \mathrm{N}, 73^{\circ} 5^{\prime} \mathrm{W}, 20$ Aug 1953, Langenheim
3519 (COL, MICH). Páramo de Monguí, laguna Colorada, $5^{\circ} 43^{\prime} \mathrm{N}, 72^{\circ} 50^{\prime} \mathrm{W}, 1 \mathrm{Sep}$ 1998, Calleja et al. 202 (MA). Páramo Ruso, $5^{\circ}$ $40^{\prime} \mathrm{N}, 73^{\circ} 5^{\prime} \mathrm{W}, 12$ July 1968 , Barkley $38 \mathrm{C} 148$ (GH). Quebrada de Becerra, NW de Duitama, $5^{\circ} 40^{\prime} \mathrm{N}, 73^{\circ} 5^{\prime} \mathrm{W}, 4$ Aug 1940, Cuatrecasas 10391 (F). Toca, vereda La Colorada, páramo de Santo Ecce Homo, $5^{\circ} 34^{\prime}$ N, $73^{\circ} 12^{\prime}$ W, 24 Apr 1982, Bejarano 208 (COL). Tutasa, km 10 of the road to El Páramo, $6^{\circ} 2^{\prime} \mathrm{N}, 72^{\circ} 51^{\prime} \mathrm{W}$, 23 Feb 1999, Stancik \& Medina 2430 (COL). Upper rio Surba, $15 \mathrm{NW}$ of Duitama, $5^{\circ} 55^{\prime} \mathrm{N}$, $73^{\circ} 7^{\prime}$ W, 7 May 1944, Fosberg 21901 (NY). Villa de Leyva, $5^{\circ} 37^{\prime} \mathrm{N}, 73^{\circ} 33^{\prime} \mathrm{W}, 20$ July 1979, Melampy 244 (MO). Santuario de Fauna y Flora de Iguaque, $5^{\circ} 37^{\prime} \mathrm{N}, 73^{\circ} 33^{\prime} \mathrm{W}$, Piragua et al. 28 (COL). Villa de Leyva, Santuario de Iguaque, $5^{\circ} 37^{\prime} \mathrm{N}, 73^{\circ} 33^{\prime} \mathrm{W}$, 20 July 1979, Melampy 242 (COL). CundinAMARCA: Villapinzón, $5^{\circ} 13^{\prime} \mathrm{N}, 73^{\circ} 31^{\prime} \mathrm{W}$, May 1998, Cortés \& Sandra 1608 (COL), Fernández et al. 15557 (MA), Fernández et al. 15565 (MA).

Etymology. Named in honor of José Luis Fernández Alonso, co-collector of the type, as well as some paratypes.

Discussion. Geranium alonsoi is an endemic to Central Colombia. Of the Geranium that grow in Colombian paramos, it resembles most closely G. sibbaldioides subsp. elongatum (Wedd.) Aedo, from which it is easily distinguished by its longer cymules which much overlap adjacent leaves. Cymules of G. alonsoi present a well developed peduncle, a pair of bracteoles, and a pedicel, while in G. sibbaldioides subsp. elongatum cymules are restricted to a short pedicel. Additionally, leaves of $G$. alonsoi are glabrous or with scattered hairs on abaxial side and margin, and less deeply divided those of G. sibbaldioides subsp. elongatum, which has abundant hairs at least on abaxial side. Fruit of G. alonsoi is shorter than in G. sibbaldioides subsp. elongatum.

Geranium costaricense also resembles $G$. alonsoi. Upon closer inspection, however, the difference between them become apparent, with leaf segments 3-9-lobed at apex, not deeply divided, short cymules (ratio cymule length/leaf length $=0.4-1.4$ ), and a rostrum with a narrowed apex in $G$. costaricense versus leaf segments 1(3)-lobed at apex, deeply divided, long cymules (ratio cymule length/leaf length $=(1.9) 2.6-4.2)$, and a rostrum without 


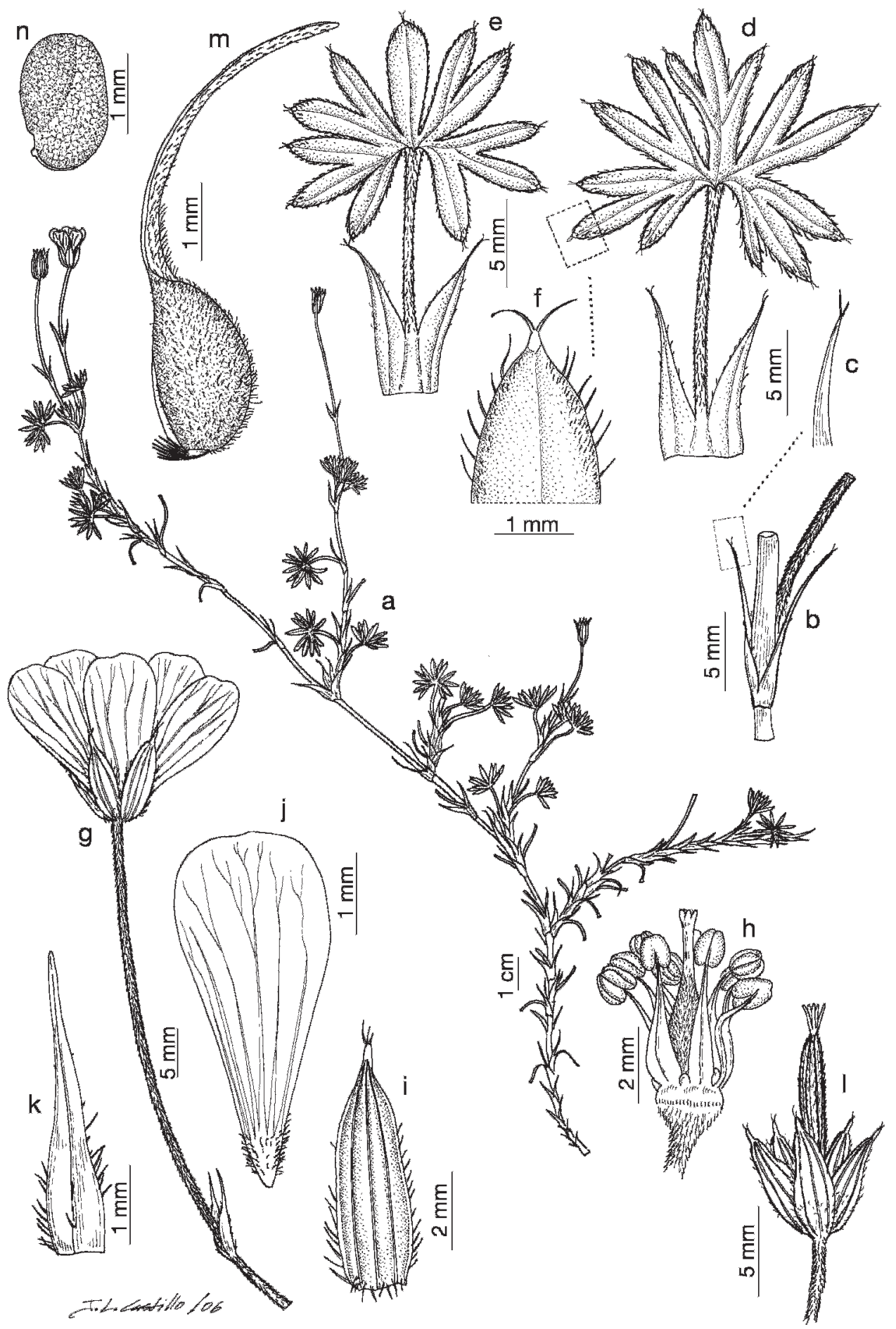

Fig. 1. Geranium alonsoi. a. Habit. b. Stipules. c. Stipule apex. d-e. Leaves. f. Leaf lobe apex. g. Cymule. h. Flower without petals and sepals. i. Sepal. j. Petal. k. Staminal filament. l. Fruit. m. Mericarp. n. Seed. (ac, e-f: from Langenheim 3519, MICH; d, j: from Barclay 7582, COL; g, i, k-1: from Bejarano 208, COL; m, n: from Fernández Alonso et al. 15557, MA). 


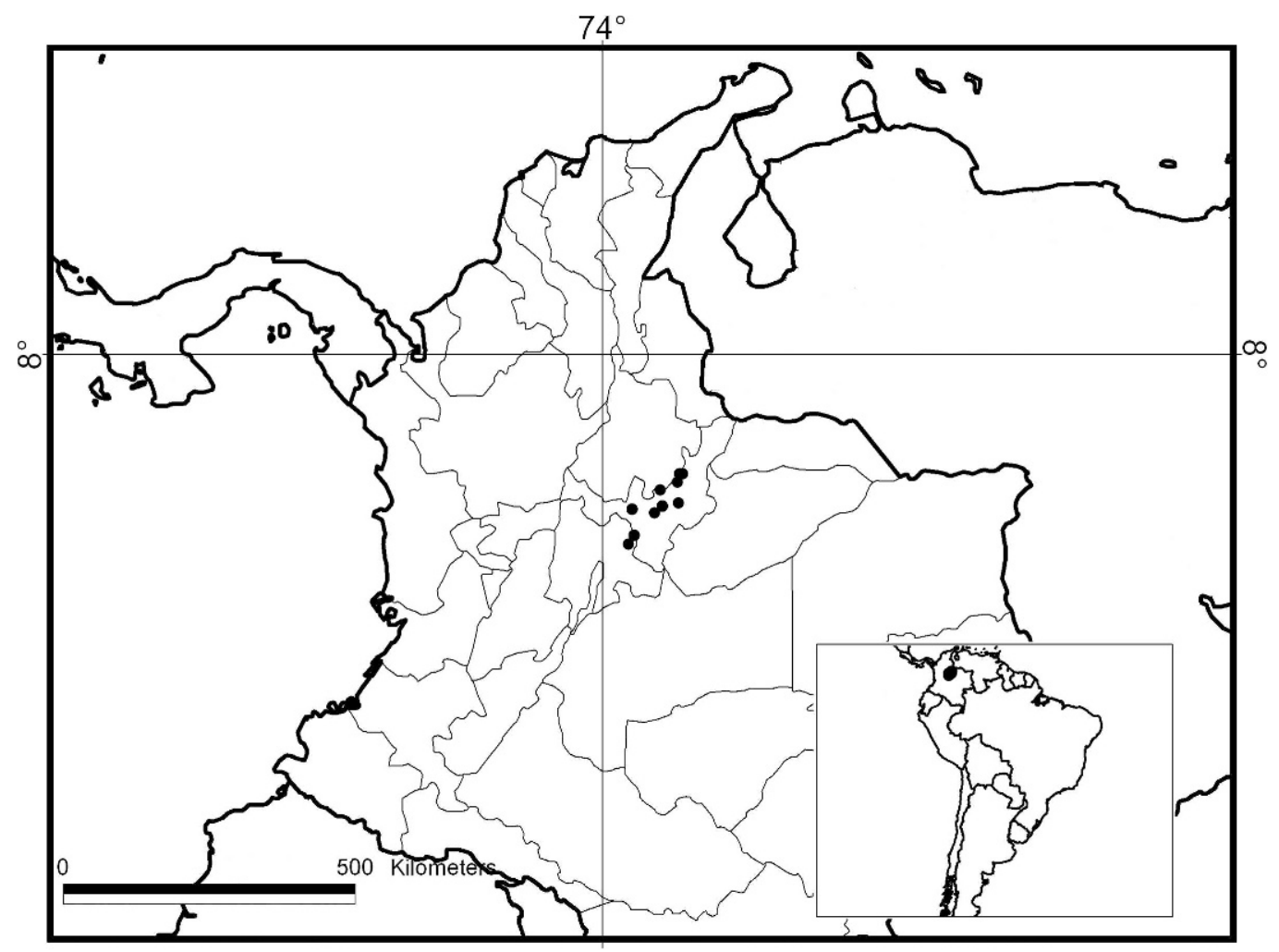

$74^{\circ}$

FIG. 2. Distribution of Geranium alonsoi Aedo.

narrowed apex in G. alonsoi. In addition, the geographical ranges of $G$. costaricense and $G$. alonsoi do not overlap at all: the first species is endemic to Costa Rica and Panama.
Species of Geranium sect. Neoandina known from Colombia, and more or less sympatric with $G$. alonsoi, are indicated in the key by an asterisk in order to facilitate identifications.

\section{Key to the Species of GeraniUm SeCt. NEOANDINA AND SECT. AZORELLOIDA}

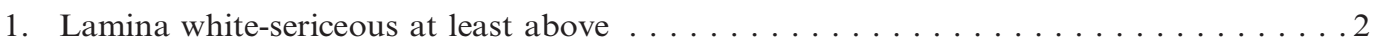

2. Lamina sericeous above, glabrous beneath

G. humboldtii Spreng.

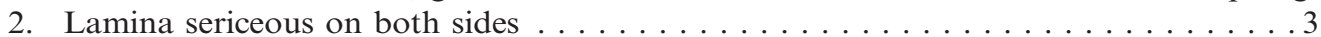

3. Lamina digitate, with 3-5 segments; lateral segments upward . . . . . . . .

G. digitatum R. Knuth

3. Lamina palmatifid to palmatisect; lateral segments patent or downward . . . . . 4

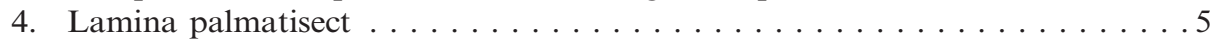

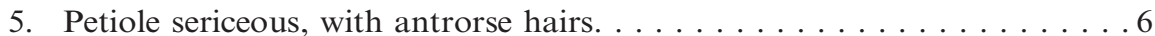

6. Petals $4-7 \mathrm{~mm}$; nectaries glabrous; fruit rostrum without a narrowed apex ................. G. tovarii Aedo

6. Petals $11-15 \mathrm{~mm}$; nectaries hairy; fruit rostrum with a narrowed apex $1-1.5 \mathrm{~mm}$ long . . . . . . . . . . . G. weddellii Briq.

5. Petiole sericeous, with patent or retrorse hairs . . . . . . . . . .

7. Lamina $0.45-0.82 \mathrm{~cm}$ long; petioles with retrorse, \pm appressed hairs $0.2-0.3 \mathrm{~mm}$ long; sepals $4.5-7.2 \mathrm{~mm}$ long with a mucro $0.5-0.9 \mathrm{~mm}$ long . . . . . . . . . . . . . G. ecuadoriense Hieron.

7. Lamina $0.9-1.7 \mathrm{~cm}$ long; petioles with patent to retrorse (not appressed) hairs $0.8-1.6 \mathrm{~mm}$ long; sepals $7-9 \mathrm{~mm}$ long with a mucro 
ca. $0.3 \mathrm{~mm}$ long

G. sericeum Willd. ex Spreng.

4. Lamina palmatifid . .

8. Middle and usually lateral leaf segments 3-lobed . . . . . . G. ruizii Hieron.

8. Middle and lateral leaf segments entire . . . . . . . . . . . . . . 9

9. Lamina $0.2-0.5(-0.6) \mathrm{cm}$ long, with 5 segments; sepals without

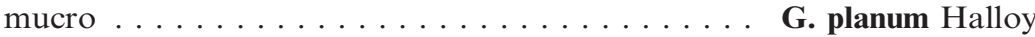

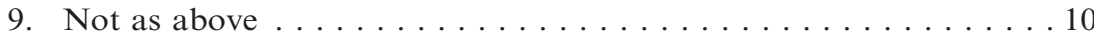

10. Petals $7-10.5 \mathrm{~mm}$ long; fruit rostrum without a narrowed apex; lamina $0.42-0.7 \mathrm{~cm}$ long. . . . . . . G. crassipes Hook. ex A. Gray

10. Petals $13-18 \mathrm{~mm}$ long; fruit rostrum with a narrowed apex; lamina $0.9-1.2 \mathrm{~cm}$ long. . . . . . . . . . . G. nivale R. Knuth

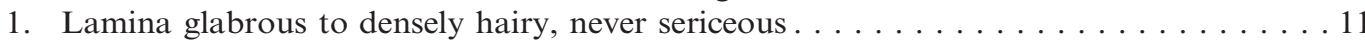

11. Lamina tridentate at the apex, with entire or rarely dentate teeth; petals

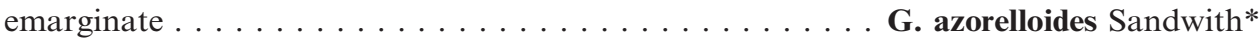

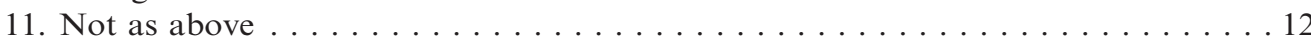

12. Lamina tripartite . . . . . . . . . . . . . G. campii H.E. Moore

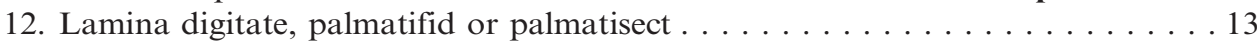

13. Lamina digitate, with 3-5 segments; lateral segments upward . . . . . . . . 14

14. Lamina glabrous or with antrorse cilia on the margin; middle leaf segment entire . . . . . . . . . . . . . G. maniculatum H.E. Moore*

14. Lamina with patent cilia on the margin; middle leaf segment 3-lobed

. . . . . . . . . . . . . . . . . . . G. rhomboidale H.E. Moore*

13. Lamina palmatifid or palmatisect; lateral segments patent or downward . . 15

15. Lamina palmatisect. . . . . . . . . . . . . . . . . . 16

16. Petiole with retrorse, appressed hairs; nectaries hairy; fruit rostrum with a narrowed apex $1 \mathrm{~mm}$ long . . . . . . . . G. macbridei Aedo

16. Petiole with patent hairs (sometimes glabrous); nectaries glabrous; fruit rostrum without a narrowed apex . . . . G. multipartitum Benth.*

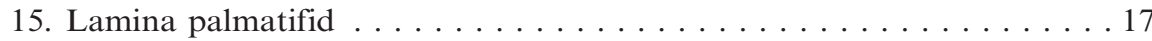

17. Stipules scarious, stramineous, obtuse

G. stramineum Triana \& Planch.*

17. Stipules papery, reddish, lanceolate. . . . . . . . . . 18

18. Petiole with antrorse hairs . . . . . . . G. jaekelae J.F. Macbr.

18. Petiole with patent to retrorse hairs. . . . . . . . . . . 19

19. Cymules (1.9)2.6-4.2 times as long as adjacent leaves ....

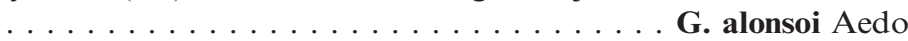

19. Cymules 0.5-1.6(1.8) times as long as adjacent leaves . . . 20

20. Lamina hairy on one or both sides . . . . . . . . 21

21. Stipules not ending in bristle .... G. pavonianum Briq.

21. Stipules ending in $1-3$ bristles $0.2-0.8 \mathrm{~mm}$ long . . . 22

22. Without peduncles; middle segment of the leaf with 1(3) lobes at apex .

G. sibbaldioides subsp. elongatum (Wedd.) Aedo*

22. Peduncles $8-53 \mathrm{~mm}$ long; middle segment of the leaf with (3)4-9 lobes at apex . . . . . . . . . .

. G. costaricense H.E. Moore

20. Lamina glabrous except on the margins . . . . . . . 23

23. Middle segment of the lamina entire, sometimes with a lateral tooth

G. sibbaldioides Benth. subsp. sibbaldioides*

23. Middle segment of the lamina 3-9-lobed at the apex . 24

24. Petiole with patent, eglandular hairs $0.2-0.7 \mathrm{~mm}$ long; petals $15-18 \mathrm{~mm}$ long ... . G. foreroi Aedo*

24. Petiole with retrorse, appressed, eglandular hairs $0.1-0.4(0.7) \mathrm{mm}$ long; petals $8.1-15.5 \mathrm{~mm}$ long. . 25 
25. Lamina (1.7)1.9-2.8 $\mathrm{cm}$ wide, deeply divided (middle segment 3-9-lobed at the apex; ratio second sinus / middle-segment length $=$ 0.33-0.53); rostrum 9-9.5 $\mathrm{mm}$ long, with a narrowed apex $0.5-1 \mathrm{~mm}$ long. . . . . . . . . . . . . . . . . G. costaricense H.E. Moore

25. Lamina $0.8-1.85 \mathrm{~cm}$ wide, shallowly divided (middle segment 3-lobed at the apex; ratio second sinus / middle-segment length $=0.1-$ 0.38); rostrum 5-9 $\mathrm{mm}$ long, without a narrowed apex . . . . . . . . . . 26

26. Sepals with a mucro $0.6-1.2 \mathrm{~mm}$ long; petals $8.1-13.2 \mathrm{~mm}$ long, usually glabrous . . . . . . . . . . . . . .

. . . G. sibbaldioides subsp. beckianum Aedo

26. Sepals with a mucro $0.3-0.7 \mathrm{~mm}$ long; petals $10.5-21 \mathrm{~mm}$ long . . . . . . . . 27

27. Petals hairy on both sides, mainly on the base of adaxial side; nectaries glabrous ... . . G. paludosum R. Knuth*

27. Petals glabrous; nectaries hairy .... $\ldots \ldots \ldots$. . . . sagasteguii Aedo

\section{Literature Cited}

Aedo, C. 2000. The genus Geranium L. (Geraniaceae) in North America. I. Annual species. Anales Jard. Bot. Madrid 58: 39-82.

Aedo, C. 2001a. Taxonomic revision of Geranium sect. Brasiliensia (Geraniaceae) Syst. Bot. 26: 205-215.

Aedo, C. 2001b. The genus Geranium L. (Geraniaceae) in North America. II. Perennial species. Anales Jard. Bot. Madrid 59: 3-65.

Aedo, C. 2004. A new species of Geranium sect. Neoandina (Geraniaceae) from Peru. Harvard Papers Bot. 9: 1-4.

Aedo, C., F. Muñoz Garmendia, and F. Pando. 1998. World checklist of Geranium L. (Geraniaceae). Anales Jard. Bot. Madrid 56: 211-252.
Aedo, C., J. J. Aldasoro, And C. Navarro. 2002. Revision of Geranium sections Azorelloida, Neoandina, and Paramensia (Geraniaceae). Blumea 47: 205-297.

Aedo, C., J. J. Aldasoro, L. Sáez, and C. Navarro. 2003. Taxonomic revision of Geranium sect. Gracilia (Geraniaceae). Brittonia 55: 93-126.

Aedo, C., C. Navarro, and M. L. Alarcón. 2005. Taxonomic revision of Geranium sections Andina and Chilensia (Geraniaceae). Bot. J. Linn. Soc. 149: 1-68.

KNUTH, R. 1912. Geranium L., p. 43-221, 575-583. In A. Engler [ed.], Das Pflanzenreich IV.129 (Heft 53). Engelmann, Leipzig.

Moore, H. E. 1943. A revision of the genus Geranium in Mexico and Central America. Contr. Gray Herb. 146: 1-108, 5 "Plate". 